\title{
Band Structure of New ReFeAsO Superconductors
}

\author{
Sergei P. Kruchinin ${ }^{1}$, Arkadiy A. Zolotovsky ${ }^{2}$, Hyun-Tak Kim ${ }^{3}$ \\ ${ }^{1}$ Bogolyubov Institute for Theoretical Physics, NASU, Kiev, Ukraine \\ ${ }^{2}$ Lashkaryov Institute of Semiconductor Physics, Kiev, Ukraine \\ ${ }^{3}$ Metal-Insulator Transition Laboratory, Electronics and Telecommunications Research Institute, \\ Daejeon, South Korea \\ Email: skruchin@i.com.ua
}

Received March 19, 2013; revised April 21, 2013; accepted May 20, 2013

Copyright (C) 2013 Sergei P. Kruchinin et al. This is an open access article distributed under the Creative Commons Attribution License, which permits unrestricted use, distribution, and reproduction in any medium, provided the original work is properly cited.

\begin{abstract}
We investigate the band structure of Fe-based superconductors using the first-principle method of density-functional theory. We calculated the band structure and the density of states at the Fermi level for ReFeAsO $(\mathrm{Re}=\mathrm{Sm}, \mathrm{Er})$ superconductors. Our calculations indicate that the maximum critical superconducting transition temperature $T_{c}$ will be observed for compounds with Sm and Er at 55 and $46 \mathrm{~K}$, respectively.
\end{abstract}

Keywords: Iron-Based Superconductors; Band Structure; Density of State; Superconducting Critical Temperature

\section{Introduction}

The discovery of a new class of high-temperature superconductors-Fe-based layered compounds-aroused a significant interest at the beginning of 2008 and gave hope for a progress in the synthesis of novel high-temperature superconductors (HTSC) up to room-temperature superconductors $[1,2]$. The important role in the explanation of physical properties of superconductors is played by calculations of the electronic structure from the first principles. It is worth noting that one of the basic properties of high-temperature superconductors is the presence of many bands [3]. In the work [4], it was shown that the formation of a superconducting state depends essentially on the density of states near the Fermi surface.

In the present work, we investigate the band structure of some Fe-based compounds, by using the first-principle method of density-functional theory. Compound

SmFeAsO showed the evidence of superconductivity at as high as $55 \mathrm{~K}$. Also the superconducting ErFeAsO has recently been found [5].

These superconductors have a complicated multiband structure. We calculated the band structure and the density of states at the Fermi level. Our calculations indicate that the maximum critical superconducting transition temperature $T_{c}$ will be observed for compounds with Sm. We obtain that $T_{c}=46 \mathrm{~K}$ for the compound ErFeAsO.

The novelty of this work is that we are having band calculations from first principles and using a magnetic pairing mechanism makes the assessment of the super- conducting transition temperature $T_{c}$. We first present the band calculations of superconductor ErFeAsO, which isopened much later than other iron superconductors.

\section{Methodology}

We calculate the band structures of HTSC, by using the Band Lab program. This program is designed for Windows computer systems and performs calculations of the electronic structure of crystalline solids.

The bands are calculated using the method outlined by Pickett [6] within the density-functional scheme [7]. Since a summary of the method and its applications has been presented by Devreese and Van Camp [8], we outline the method here only in brief.

Starting with the $\mathrm{N}$-electron problem in the field of ionic potentials

$$
V_{\text {ion }}(r)=\sum_{m} v_{\text {ion }}\left(\boldsymbol{r}-\boldsymbol{R}_{\boldsymbol{m}}\right) .
$$

with the ions at static positions $\boldsymbol{R}_{\boldsymbol{m}}$, we have the Hamiltonian

$$
\begin{aligned}
H= & \sum_{i} p_{i}^{2} / 2 m+\int n(r) V_{\text {ion }}(r) \mathrm{d} r \\
& +\frac{1}{2} \iint n(r) v\left(r-r^{\prime}\right) n\left(r^{\prime}\right) \mathrm{d} r \mathrm{~d} r^{\prime} .
\end{aligned}
$$

Here, $n(r)$ is the electron density operator at $r$, and $v\left(r-r^{\prime}\right)$ is the Coulomb interaction.

Hohenberg and Kohn established that there is a one- 
to-one correspondence between the ionic potential $V_{\text {ion }}$ (to within an arbitrary constant) and the ground state density $n$, so that the many-body problem can be considered as a functional of the density, rather than a functional of the potential [7].

Hohenberg and Kohn [9] established that, for variations $\delta n$ of the energy density which conserve the particle number $N$, the ground-state energy functional $E[n]$ is minimized by the ground-state density, with corrections of the order of $\delta n^{2}$. The resulting variational principle allows one to accurately obtain numerical results.

The energy functional has the form:

$$
\begin{aligned}
E[n]= & T_{0}[n]+\int V_{\text {ion }}(r) n(r) \mathrm{d} r \\
& +\frac{1}{2} \iint n(r) v\left(r-r^{\prime}\right) n\left(r^{\prime}\right) \mathrm{d} r \mathrm{~d} r^{\prime}+E_{x c}[n],
\end{aligned}
$$

where $T_{0}[n]$ is the kinetic energy of the system of interacting electrons with the density $n(r)$, and $E_{x c}[n]$ is the exchange-correlation energy. If one has adequate approximations for $T_{0}[n]$ and $E_{x c}[n]$, the energy can be obtained directly from relation (3), by the minimization with respect to $n(r)$.

The energy functional is minimized by the density functional described by the above-presented relations, by performing iterations to attain the self-consistency.

Within the density-functional theory, the one-electron eigenvalues and eigenfunctions have no physical meaning and are not physically realizable. Some reviews containing discussions of applications of the density-functional theory, as well as the generalization to spin-polarized systems, are given, for example, in [10].

\section{Band Structures of a Number of Oxypnictides ReFeAsO}

The crystal structure of compound ReFeAsO is shown in Figure 1. Such substances possess the tetragonal structure at room temperature. The crystal structure is formed by alternating FeAs layers revealing antiferromagnetism which are separated by ReO layers. The results of calculations of the electronic spectrum can be found in [11]. It should be emphasized that the specific features of the electronic spectrum are related to the quasi-two-dimensional character of superconductivity in FeAs planes.

In many works, the increase in $T_{c}$ which is caused by the replacement of $\mathrm{La}$ by other rare-earth elements is frequently named the "chemical” pressure [11]. A similar effect is characteristic of high-temperature superconductors [12]. The substitution of various rare-earth ions into the series of compounds ReFeAsO, as distinct from cuprate superconductors $\mathrm{ReBaCuO}$, leads to a quite large dispersion of values of $T_{c}$. We may consider the magnetic mechanism of pairing in Fe-based superconductors. Spins of rare-earth ions interact strongly with spins of

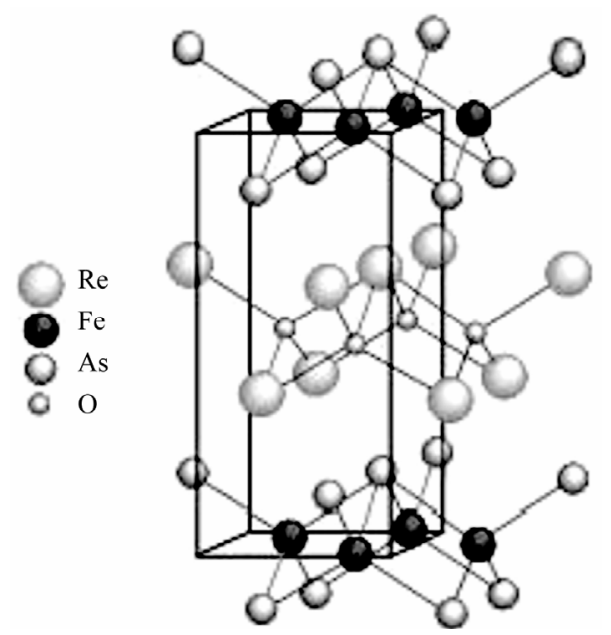

Figure 1. Crystalline structure of ReFeAsO.

iron and can essentially influence the spectrum of spin fluctuations in FeAS planes, which increases $T_{c}$ if the magnetic mechanism of pairing is dominant.

We have carried out $a b$ initio calculations for a number of oxyphictides ReFeAsO in the framework of an approach based on density-functional theory. The results of calculations are presented in Figures $\mathbf{2}$ and $\mathbf{3}$ for the band structure and the density of states in ReFeAsO. At a comprehensive study of the density of states, the relationship of the density of states at the Fermi level and the value of $T_{c}$ is revealed. When comparing the band calculations Figures $\mathbf{2}$ and $\mathbf{3}$ shows that the density of states at the Fermi level is much higher in Figure 2 than in Figure 3, that is a superconductor $\mathrm{SmFe} \mathrm{AsO}$ has a higher density of states than ErFeAsO. Therefore, the estimates for formula (4) give the above superconducting transition temperature for SmFe AsO. In Figure 4, we give the results of our theoretical calculations (circles) and experimental values (squares) of the critical supercondicting transition temperature. We indicate a good agreement with experimental data. In the calculations of $T_{c}$, we used the BCS-like formula for the magnetic mechanism [13]

$$
T_{C}=1.14 \theta \exp \left(\frac{1}{N\left(E_{f}\right) V}\right),
$$

where $\theta$-energy of spin fluctuations, $V$-attractive pairing potential acting between electrons, $N\left(E_{f}\right)$-density of states on the Fermi surface.

\section{Summary}

The discovery of a new type of high-temperature superconductivity in a FeAs system generates expectation of the appearance of novel superconductors with higher $T_{c}$ by means of the doping that consists of the substitution of the atoms lying outside of the principal layers comprising FeAs. In FeAs, such a doping is favorable due to 

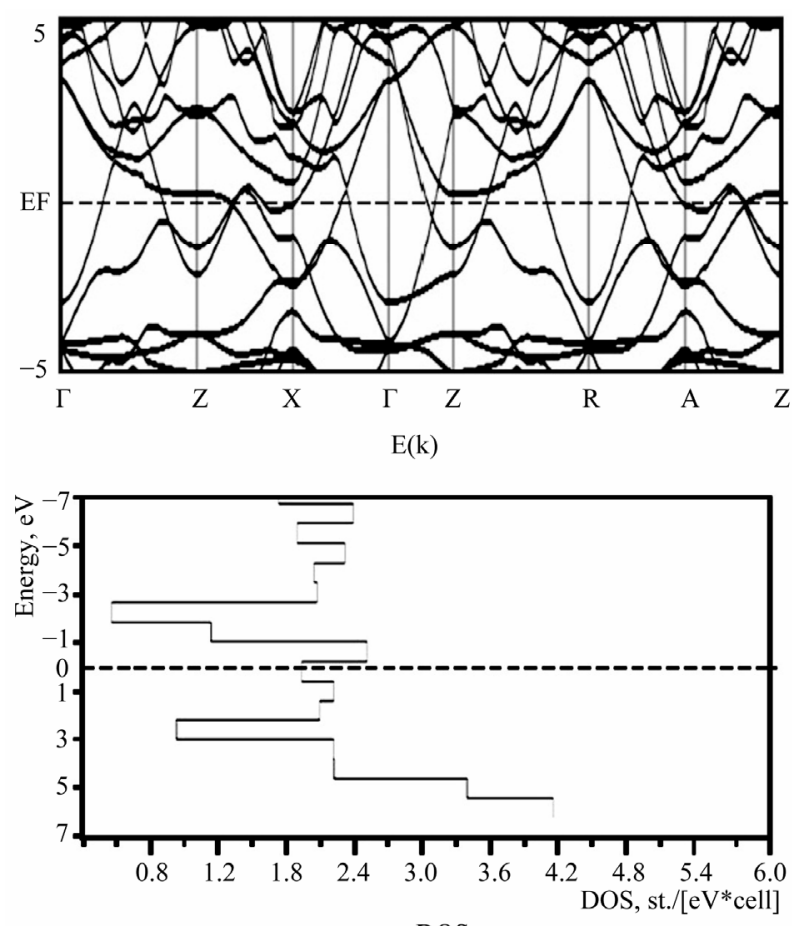

DOS

Figure 2. Results of our calculations of the band structure and the density of states for $\mathrm{SmFeAsO}$ with $T_{c}=55.0 \mathrm{~K}$.
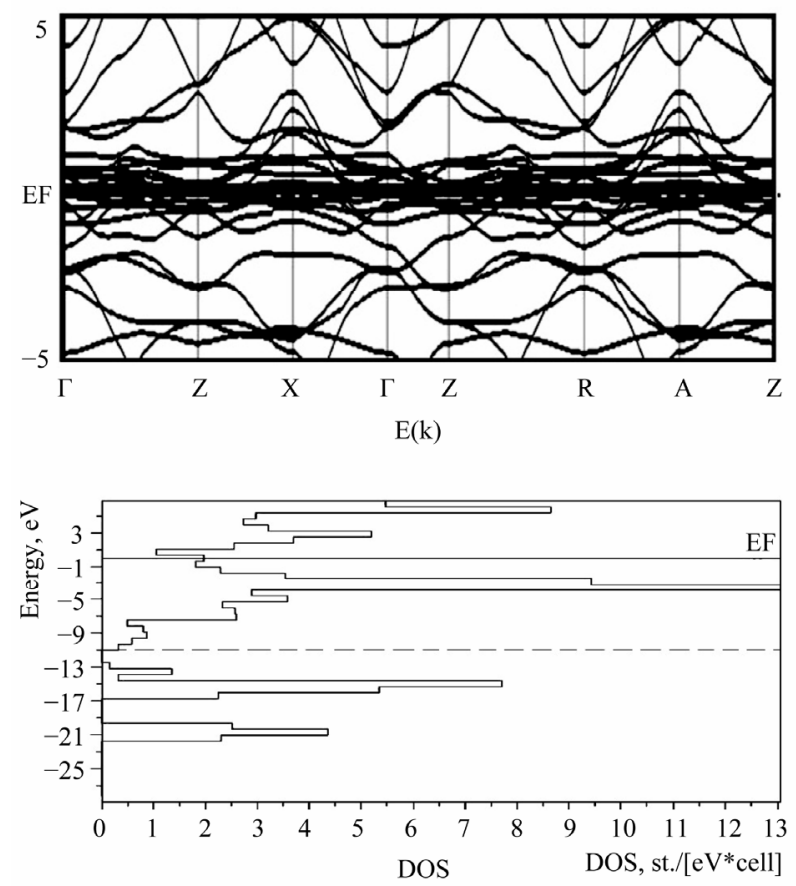

Figure 3. Results of our calculations of the band structure and the density of states for ErFeAsO with $T_{c}=46 \mathrm{~K}$.

the easy replacement of chemical elements. In compounds ReFeAsO, the substitution of the rare-earth element is of special interest. We have carried out $a b$ initio calculations for a number of oxypnictides ReFeAsO in

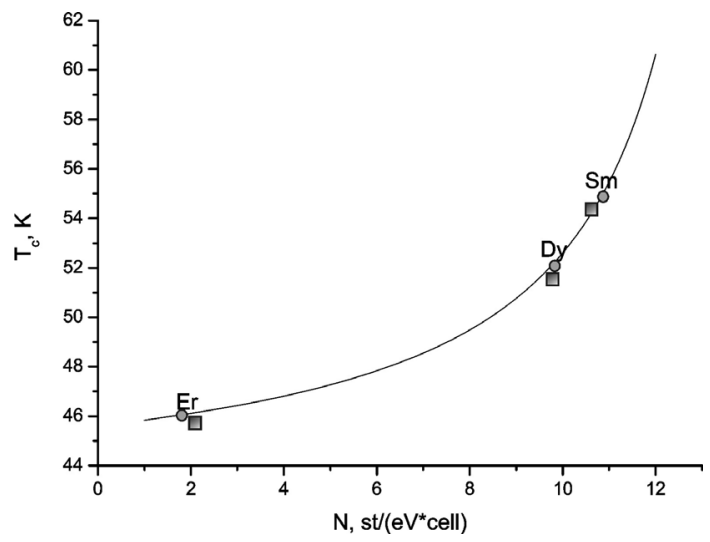

Figure 4. Dependence of $T_{c}$ on the density of states on the Fermi surface $N\left(E_{f}\right)$ for $\operatorname{ReFeAsO}[13]$ ( $\bigcirc$ theory, $\square-\mathrm{ex}-$ periment).

the framework of density-functional theory. Our results indicate that the maximum critical superconducting transition temperature will be attained for compounds with $\mathrm{Sm}\left(T_{c}=55 \mathrm{~K}\right)$ and for ErFeAsO $\left(T_{c}=46 \mathrm{~K}\right)$.

\section{Acknowledgements}

The authors thank Profs. Yu. Matsuda and K. Bennemann for their continued encouragement and helpful discussions. This paper is supported by Korea-Ukraine project of MEST in Korea, and MIT Creative Research Project in ETRI.

\section{REFERENCES}

[1] Y. Kamahira, T. Watanabe, M. Hirano and H. Hasono, Journal of the American Chemical Society, Vol. 139, 2008, pp. 3296-3297. doi:10.1021/ja800073m

[2] S. Kruchinin, H. Nagao and S. Aono, "Modern Aspects of Superconductivity: Theory of Superconductivity,” World Scientific, Singapore, 2010, p. 232.

[3] H. Nagao, S. P. Kruchinin, A. M. Yaremko and K. Yamaguchi, International Journal of Modern Physics B, Vol. 16, 2002, pp. 3419-3428. doi:10.1142/S0217979202012220

[4] S. P. Kruchinin and A. M. Yaremko, Superconductor Science and Technology, Vol. 11, 1998, pp. 4-8.

[5] P. Shirage, K. Miyazawa, K. Kihou, C.-H. Lee, H. Kito, K. Tokiwa, et al., Europhysics Letters, Vol. 92, 2010, pp. 57011-57015.

[6] S. P. Kruchinin and H. Nagao, Physics of Particles and Nuclei, Vol. 36, 2005, pp. 127-130.

[7] W. E. Pickett, Reviews of Modern Physics, Vol. 61, 1989, pp. 433-512. doi:10.1103/RevModPhys.61.433

[8] J. T. Devreese and P. Van Camp, Eds., "Electronic Structure, Dynamics, and Quantum Structural Properties of Condensed Matter,” Plenum, New York, 1985. doi:10.1007/978-1-4757-0899-8

[9] P. Hohenberg and W. Kohn, Physical Review B, Vol. 136, 
1964, pp. 864-871. doi:10.1103/PhysRev.136.B864

[10] W. Kohn and L. J. Sham, Physical Review A, Vol. 140, 1965, pp. 1133-1138.

[11] Y. Mizuguchi, et al., Superconductor Science and Technology, Vol. 23, 2010, pp. 054013-0540018. doi:10.1088/0953-2048/23/5/054013
[12] A. S. Davydov and S. P. Kruchinin, Physics C, Vol. 179, 1991, pp. 461-468. doi:10.1016/0921-4534(91)92196-I

[13] P. Monthoux, A. Balatsky and D. Pines, Physical Review $B$, Vol. 46, 1992, pp. 14803-14812. doi:10.1103/PhysRevB.46.14803 